Quim. Nova, Vol. 34, No. 7, 1224-1230, 2011

\title{
ANÁLISE TÉRMICA APLICADA A FÁRMACOS E FORMULAÇÕES FARMACÊUTICAS NA INDÚSTRIA FARMACÊUTICA
}

\author{
Marcelo Antonio de Oliveira* \\ Centro Universitário Norte do Espírito Santo, Universidade Federal do Espírito Santo, Rod. BR 101 Norte, km 60, 29932-540 \\ São Mateus - ES, Brasil \\ Maria Irene Yoshida e Elionai Cassiana de Lima Gomes \\ Departamento de Química, Universidade Federal de Minas Gerais, Av. Antônio Carlos, 6627, 31270-901 Belo Horizonte - MG, Brasil
}

Recebido em 16/10/10; aceito em 11/2/11; publicado na web em 15/4/11

\begin{abstract}
THERMAL ANALYSIS APPLIED TO DRUGS AND PHARMACEUTICAL FORMULATIONS IN PHARMACEUTICAL INDUSTRY. Several matters of the pharmaceutical demonstrate the great importance of thermal analysis application, especially TG and DSC for the pharmaceutical industry future, namely: characterization of the drugs with the thermal events definition, in studies of drug purity, in the polymorphs identification, in compatibility studies of solid dosage pharmaceutical formulations, in drugs and pharmaceutical formulations thermal stability, and in determination of shelf life for isothermal degradation kinetics by extrapolation using the Arrhenius equation. Thus, the test results obtained from thermal analysis are directly related to the quality of a pharmaceutical product, whether the stability or bioavailability of the pharmaceutical product.
\end{abstract}

Keywords: thermal analysis; pharmaceutical industry; characterization.

\section{INTRODUÇÃO}

Vários estudos relacionados à aplicação da termogravimetria (TG) e calorimetria exploratória diferencial (DSC) na caracterização, avaliação de pureza, compatibilidade de formulação farmacêutica, identificação de polimorfismo, estabilidade e decomposição térmica de fármacos e medicamentos encontram-se descritos na literatura. A TGé utilizada para medir a variação de massa em função da temperatura em uma atmosfera controlada sob um programa de aquecimento. Para fins farmacêuticos, seu uso é descrito na caracterização, determinação de pureza e de umidade, identificação de pseudopolimorfismo, na avaliação da estabilidade de fármacos e medicamentos e em estudos de cinética de degradação. A DSC é utilizada para medir a diferença de fluxo de calor entre uma substância e um material de referência em função de um programa de aquecimento ou resfriamento. $\mathrm{Na}$ área farmacêutica é utilizada na caracterização térmica e determinação da pureza de fármacos, estudos de compatibilidade entre os constituintes da formulação e identificação de polimorfismo com determinação das entalpias de cada forma cristalina.

Recentemente, muitos trabalhos em análise térmica têm sido publicados na área aplicada à indústria farmacêutica. Desta forma, as indústrias brasileiras estão, cada vez mais, demonstrando o interesse neste âmbito. Os artigos publicados demonstram que os dados obtidos por análise térmica estão diretamente relacionados com a qualidade final de um produto farmacêutico, seja quanto à eficácia terapêutica do medicamento ou à estabilidade do mesmo ao longo do prazo de validade. Além disso, muitos órgãos regulamentadores de insumos e produtos farmacêuticos já descrevem a importância da análise térmica e dos parâmetros de qualidade dela provenientes. ${ }^{1-7}$

\section{APLICAÇÕES}

A Farmacopeia Japonesa, JP XIV, foi a primeira a descrever os

\footnotetext{
*e-mail: oliveirama.ufes@gmail.com
}

métodos de análise térmica, mas sem nenhuma exigência em monografias. ${ }^{6}$ A TG foi descrita apenas como um método alternativo para o teste de perda por dessecação em matérias-primas e ainda foi citada como suporte para aplicações do tipo estudo de pureza, estabilidade e decomposição térmica. Já o DSC foi citado para as aplicações como ponto de fusão, calor de fusão, transição cristalina e polimorfismo. As Farmacopeias Americana, USP 31, Britânica, BP 2007, e a Europeia, EP 2007, também já apresentam o mesmo texto descrito pela JP XIV, definindo os termos TG e DSC e suas aplicações, e ainda acrescentam citações de aplicação do DSC para estudos de pureza utilizando a Equação de van’t Hoff e de compatibilidade de formulações farmacêuticas. ${ }^{1,3,6,7} \mathrm{Na}$ Farmacopeia Portuguesa estão definidos somente os termos para TG, mas não há indicação clara de sua utilização, nem relatos da técnica de DSC. ${ }^{4}$ Dentre as farmacopeias, o único código oficial que preconiza o uso da análise térmica em monografias é a USP 31, que apresenta exigência para determinação de umidade por TG para os seguintes fármacos: cloridrato de amilorida, azitromicina, mesilato de bromocriptina, imipenem, paricalcitol, repaglinida, sulfato de vinblastina e sulfato de vincristina.

Vários livros tratam especificamente do uso da análise térmica no campo das Ciências Farmacêuticas, utilizando a técnica na caracterização, estudos de pureza, estabilidade e compatibilidade, polimorfismo e cinética de degradação térmica; mas muito pouco é publicado nesta área apesar da importância desta ferramenta analítica. ${ }^{8-13}$

Alguns artigos descrevem a utilização prática da análise térmica para cada aplicação prevista.

\section{Caracterização}

$\mathrm{Na}$ caracterização de fármacos e produtos farmacêuticos é possível identificar e quantificar os eventos de perdas de massas, e identificar os processos endotérmicos ou exotérmicos; Rustichelli et al., McGregor et al., Rodrigues-Spong et al. e Porter III et al. 
relataram sobre a carbamazepina; Giordano et al. relata sobre o diclofenaco de sódio. ${ }^{14-18}$

Rustichelli et al. caracterizaram polimorfos da carbamazepina utilizando DSC combinada com outras técnicas analíticas. De acordo com o polimorfo utilizado no medicamento, a carbamazepina, que é um fármaco anticonvulsivante, pode sofrer alteração nas propriedades de dissolução e biodisponibilidade de comprimidos. As Farmacopeias Britânica e Europeia apresentam especificações incoerentes, visto que o espectro de infravermelho indicado é referente ao polimorfo da forma II e o ponto de fusão é correspondente ao da forma I. As farmacopeias também não especificam parâmetros importantes como razão de aquecimento, o que inviabiliza a caracterização, de modo homogêneo, da carbamazepina por análise térmica. ${ }^{14}$

McGregor et al. estabeleceram parâmetros para determinar a entalpia de fusão da forma III da carbamazepina e propuseram um método de quantificação para as formas I e III em mistura. No entanto, o método não pode ser usado para determinação de pureza dos polimorfos já que foi observado que, em misturas, a forma I catalisa a cristalização da forma III. ${ }^{15}$

Rodrigues-Spong et al. caracterizaram quatro polimorfos e seis cocristais para a carbamazepina utilizando DSC e infravermelho (IV). Já Porter III et al. caracterizaram duas formas polimórficas do cocristal carbamazepina-sacarina utilizando DSC, IV e DRX.16,17

Giordano et al. relataram que o uso da temperatura de fusão do diclofenaco de sódio como parâmetro de pureza, como estabelecido pelas farmacopeias não é adequado, visto que seu processo de decomposição é complexo. O diclofenaco de sódio sofre decomposição e/ou ciclização formando 1-(2,6-diclorofenil)-indolim-2-1 antes de alcançar sua temperatura de fusão, dependendo da razão de aquecimento e atmosfera do ensaio, o que acarreta em erros na determinação do ponto de fusão pelos métodos tradicionais da farmacopeia. A literatura (The Merck Index, 2000) relata fusão em $160{ }^{\circ} \mathrm{C}$, enquanto os ensaios em atmosfera inerte de nitrogênio demonstram fusão numa faixa mais adequada, em torno de $180^{\circ} \mathrm{C} .{ }^{18,19}$

\section{Determinação de umidade}

A utilização da análise térmica na determinação de umidade é descrita por Giron, comparando o método oficial das farmacopeias, Karl Fischer, com os resultados encontrados por TG. A autora conclui que não existem diferenças significativas entre os dois tipos de métodos de determinação de umidade e que a TG poderia substituir o método de Karl Fischer. ${ }^{20}$

\section{Pureza}

Quanto à determinação de pureza de um fármaco, o comitê da Organização Mundial de Saúde - OMS - recomenda o uso dos métodos de análise e especificações presentes nas farmacopeias oficiais, enquanto que para princípios ativos não farmacopeicos, recomenda-se determinar a pureza por um método absoluto como a DSC. ${ }^{21} \mathrm{~A}$ avaliação da pureza por DSC pode ser realizada pelo simples acompanhamento visual da curva DSC, observando a presença dos eventos térmicos característicos do fármaco, ou utilizando uma determinação quantitativa pelo método da Equação de van't Hoff, que determina a pureza a partir do pico de fusão do analito.

Schnitzler et al. relataram a caracterização e avaliação de pureza do acetato de dexametasona utilizando DSC na avaliação de matérias-primas, demonstrando que, em alguns casos, uma simples análise visual da curva de DSC pode indicar a presença da fusão de um contaminante. ${ }^{22}$

Ferguson et al., Macedo e Nascimento e Giron descrevem a utilização da Equação de van't Hoff para determinação de pureza de um fármaco com análise por DSC. ${ }^{23-25}$ A pureza da zidovudina com análise por DSC foi relatada por Rodrigues et al. com a utilização da Equação de van’t Hoff. ${ }^{26}$ Macedo et al. compararam os métodos oficiais de determinação de pureza estipulados nas farmacopeias para os fármacos anti-hipertensivos captopril, propranolol e nifedipina, sendo estes por titulação e espectrofotometria UV/VIS, com a DSC utilizando a Equação de van't Hoff e demonstraram não haver diferenças estatísticas entre eles. ${ }^{27}$

$\mathrm{Na}$ avaliação de pureza absoluta pelo método da Equação de van't Hoff, sabe-se que quanto maior a concentração de impurezas na amostra, menor é o ponto de fusão e mais larga é a faixa de fusão. Os dados obtidos numa curva de DSC referem-se à faixa de fusão e o calor de fusão do analito $(\Delta \mathrm{Hf})$ e a interpretação da pureza por DSC baseiam-se em uma modificação da Equação 1 de van’t Hoff:

$$
T_{s}=T_{o}-\frac{R T_{\mathrm{o}}^{2} X_{1}}{\Delta H_{f}} \times \frac{1}{F}
$$

onde: $\mathrm{T}_{\mathrm{s}}=$ temperatura da $\operatorname{amostra}(\mathrm{K}) ; \mathrm{T}_{\mathrm{o}}=$ temperatura de fusão teórica do analito puro $(\mathrm{K}) ; \mathrm{R}=$ constante universal dos gases $(8,314$ $\left.\mathrm{J} \mathrm{mol}^{-1} \mathrm{~K}^{-1}\right) ; \mathrm{X}_{1}=$ fração molar da impureza; $\Delta \mathrm{H}_{\mathrm{f}}=$ calor de fusão do componente principal puro $\left(\mathrm{J} \mathrm{mol}^{-1}\right)$ e $\mathrm{F}=$ fração da amostra que fundiu em $\mathrm{T}_{\mathrm{s}}$

Aplicando a Equação de van't Hoff, determinam-se alguns parâmetros de pureza, como a fração molar de impureza (X1) e o ponto de fusão teórico do analito puro (To), determinando a pureza absoluta ao final do processo. .,9,11,23,28,29 $^{8}$

\section{Compatibilidade de formulações farmacêuticas}

O estudo do prazo de validade, chamado de estudo de estabilidade, é um dos principais fatores avaliados no desenvolvimento de formulações farmacêuticas. Estes estudos são realizados rotineiramente pela indústria farmacêutica, porém, requerem longos períodos de armazenamento das amostras, sob condições controladas de temperatura e umidade. ${ }^{30}$ Embora não substituam os estudos convencionais, as técnicas termoanalíticas, como DSC, mostram-se extremamente úteis em estudos de estabilidade, possibilitando a escolha das formulações mais estáveis com extrema rapidez por meio dos estudos de compatibilidade.

\section{Estabilidade e compatibilidade}

Para avaliações de estabilidade e compatibilidade de formulações farmacêuticas, vários relatos são descritos na literatura como, por exemplo, Ceschel et al., Bazzo e Segatto Silva, Stulzer et al., Cides et al., Laszcz et al., Macedo et al., Freitas et al., Oliveira et al. e Mura et al. ${ }^{31-39}$

Ceschel et al. demonstraram por DSC a incompatibilidade entre o princípio ativo ácido acetilsalicílico (AAS) e o excipiente ácido esteárico. Na presença deste excipiente todas as curvas DSC se apresentavam alteradas em relação à curva inicial do AAS. Os outros excipientes encontrados na formulação não alteraram a curva DSC do ácido acetilsalicílico em misturas binárias, não sendo, desta forma, responsáveis pela degradação do princípio ativo. ${ }^{31}$

Bazzo e Segatto Silva relataram o estudo de compatibilidade de formulações de captopril, sugerindo a existência de interação com estearato de magnésio. Já Stulzer et al. também avaliaram formulações de comprimidos de captopril e apresentaram como incompatibilidade o excipiente estearato de magnésio; relatando ainda que o PVP não interage com o fármaco, e sim solubiliza o captopril mascarando o resultado da análise por DSC. . $^{32,33}$

Cides et al. apresentaram como incompatíveis numa formulação de glimepirida os seguintes excipientes: estearato de magnésio, 
lactose e Plasdone ${ }^{\circledast}$. Os autores apresentaram resultados de cinética de degradação isotérmica para a formulação farmacêutica, que apresentou Ea (energia de ativação) de $157 \mathrm{~kJ} \mathrm{~mol}^{-1} \mathrm{em}$ ar, e $150 \mathrm{~kJ}$ mol $^{-1}$ em nitrogênio. ${ }^{34}$

Laszcz et al. relataram por meio de análise com DSC que o estearato de magnésio é incompatível com mesilato de imatinibe numa formulação farmacêutica, um medicamento utilizado em leucemia. Quanto maior a proporção de estearato de magnésio, maior a interação fármaco-excipiente demonstrada por curvas DSC. No entanto, os autores descreveram que a interação deve ser fraca, visto que não foram observadas alterações nas análises de IV e DRX da mistura binária. ${ }^{35}$

Macedo et al. relataram que existe incompatibilidade entre a lactose e a hidroclorotiazida nas formulações dos medicamentos e que a celulose microcristalina seria uma alternativa numa substituição à lactose na formulação farmacêutica, visto que não apresenta interação com o fármaco. Os autores apresentaram resultados de cinética de degradação isotérmica, comparando o valor das constantes de velocidade de degradação (k) para justificar as conclusões encontradas. ${ }^{36}$

Freitas et al., avaliando pré-formulações de comprimidos osmóticos de atenolol por DSC, relataram que existem incompatibilidades entre o fármaco e todos os óxidos de polietileno utilizados. ${ }^{37}$

Oliveira et al. relataram a incompatibilidade entre estearato de magnésio e glibenclamida em formulações de comprimidos. O estudo foi concluído com análises por DSC e TG; relataram ainda que a Ea (energia de ativação) para o fármaco é sempre maior que para a formulação farmacêutica, o que demonstra a menor estabilidade do medicamento comparado ao fármaco. ${ }^{38}$

Mura et al. relataram a importância de se confirmar as possíveis incompatibilidades encontradas por DSC com estudos de análise termo-óptica e microscopia óptica, visto que para o ibuproxam a DSC indicava incompatibilidades de formulação que não eram confirmadas pelas outras técnicas. ${ }^{39}$

\section{Polimorfismo}

Para avaliação de polimorfismo, o comitê do órgão de harmonização internacional, ICH (International Conference on Harmonization), constituído por representantes de órgãos de regulamentação da União Europeia, Japão, Estados Unidos e representantes da indústria farmacêutica, estabeleceu protocolos para o controle da qualidade dos insumos farmacêuticos, que são publicados como guias ou leis em diversos países. O guia publicado pela agência americana Food and Drug Administration - FDA (ICH Q6A, 1999) - relata que a ausência de especificações para parâmetros de qualidade, como limite de solvente residual, polimorfismo e presença de enantiômeros deve ser justificada. São estabelecidos, ainda, os critérios para caracterizar a forma polimorfa e o grau de criticidade em relação ao produto final. As técnicas indicadas nesta caracterização são: difração de raios X, análise térmica, microscopias e espectroscopias. ${ }^{5}$

Para o medicamento genérico, o ICH definiu por meio da Guidance (2007) a importância do polimorfismo, como é caracterizado, sua influência na qualidade do produto farmacêutico e o grau de criticidade do polimorfismo no produto. O comitê da ICH também definiu, por meio da Guidance (1987), que se o fenômeno de polimorfismo for conhecido, uma instrução geral deve constar na monografia farmacopeica da seguinte forma: "a substância exibe o fenômeno de polimorfismo". 2,40

O estudo de polimorfismo com a utilização do DSC e TG para elucidar as formas polimórficas é relatado por alguns autores, como Moneghini et al.; Hassan et al.; Roux et al.; Bergese et al.; Palacio et al.; Caira et al.; Sacchetti; Leitão; Swanepoel et al. e Marciniec et al.. ${ }^{41-50}$

Moneghini et al. relataram através de análises de DSC e DRX que o fármaco atenolol, um anti-hipertensivo, se apresentou na forma cristalina com os agentes dispersores povidona e polivinilpirrolidona/ vinil acetato, e na forma amorfa com os dispersores crospovidona e eudragit E. Os resultados coincidem com aqueles de maiores taxas de dissolução do fármaco na fase amorfa. ${ }^{41}$

Hassan et al. caracterizaram três formas polimórficas de famotidina utilizando DSC, IV e DRX, enquanto Roux et al. relataram que existe alguma alteração no polimorfo B da famotidina quando submetido a condições de pressão, sendo avaliado por DSC. ${ }^{42,43}$

Bergese et al. caracterizaram duas formas polimórficas da nimesulida utilizando DSC, definindo a relação de monotropia entre os cristais e apresentando o cristal metaestável (Forma II) e mais solúvel. ${ }^{44}$

Palacio et al. relataram a caracterização de duas formas polimórficas de albuterol por IV, DRX, microscopia óptica e DSC. Os resultados de DSC comparam as temperaturas de decomposição das formas polimórficas, diferenciando as duas formas. ${ }^{45}$

Caira et al. caracterizaram duas formas polimórficas do ambroxol utilizando DSC e DRX, apresentando a relação de enantiotropia entre os cristais, sendo a Forma I, de maior ponto de fusão, a forma metaestável. ${ }^{46}$

Sacchetti caracterizou por DSC duas formas polimórficas do paracetamol, determinando ainda a temperatura de transição cristalina. ${ }^{47}$

Leitão identificou quatro formas polimórficas da terfenadina, utilizando condições de recristalização diferentes para a obtenção das formas cristalinas, sendo usados solventes e temperaturas variadas e empregando DSC e DRX para caracterização dos polimorfos. ${ }^{48}$

Swanepoel et al. relataram a caracterização de três formas polimórficas do mebendazol e demonstraram ainda que o método de dissolução apresentado pela Farmacopeia Americana, USP 31, não é adequado para diferenciar estes polimorfos visto que as três formas cristalinas apresentaram perfis de dissolução muito próximos. Os autores apresentaram um meio de dissolução alternativo, com a retirada do reagente laurilsulfato de sódio, que seria capaz de distinguir as diferenças de solubilidade entre as formas cristalinas do mebendazol. ${ }^{49}$

Marciniec et al. relataram os efeitos da irradiação gama sobre hidrocortizona, acetato de hidrocortizona, prednisolona e acetato de prednisolona. Os estudos foram conduzidos a partir de análises por DSC, DRX e microscopia óptica, e os resultados mostraram que não houve alteração polimórfica em nenhum dos fármacos avaliados. ${ }^{50}$

O polimorfismo pode ser identificado por técnicas analíticas diferentes de acordo com o código oficial farmacêutico utilizado. $\mathrm{Na}$ Farmacopeia Americana, USP 31, não são descritos nas monografias os dizeres referentes à presença de polimorfismo para os fármacos, como recomendado pelo ICH (Guidance, 1987). Seria impossível distinguir entre formas cristalinas distintas utilizando a técnica de infravermelho, pois o texto do capítulo geral da USP 31 apresenta a seguinte especificação para avaliação do fármaco: “ se os espectros obtidos com a amostra e a substância de referência apresentarem diferenças, dissolva as substâncias num volume mínimo de solvente, evapore à secura e registre novos espectros a partir dos resíduos". Na mesma farmacopeia, a presença do polimorfismo pode ser observada quando se exigem nas monografias análises referentes à DRX ou microscopia óptica. A DRX é exigida em monografia para 8 fármacos distintos, como exemplo, carbamazepina, etilsuccinato de eritromicina, indometacina e ritonavir; enquanto a microscopia óptica é exigida para 18 fármacos, tais como, amoxicilina, ampicilina, azitromicina, cloranfenicol, eritromicina, nistatina, tetraciclina e outros. Para a Farmacopeia Britânica, BP 2007, existe a informação clara da presença de polimorfismo em 121 fármacos, com os dizeres recomendados pelo ICH (Guidance, 1987): "a substância exibe polimorfismo"; a técnica analítica recomendada é a análise por infravermelho, com a descrição na monografia da seguinte forma: "infravermelho sem tratamento prévio do fármaco". Na Farmacopeia Brasileira, os di- 
zeres recomendados pelo ICH (Guidance, 1987) quanto à presença de polimorfismo estão presentes para dois fármacos, cloridrato de ranitidina e zidovudina, e a técnica analítica utilizada na distinção dos polimorfos é a espectrometria de infravermelho. ${ }^{51,52}$

Além disso, durante o processo de fabricação do medicamento, algumas etapas de procedimento podem levar a alterações polimórficas, tais como, processo de micronização ou moagem; granulação via úmida, secagem, compressão e descontaminação microbiológica com irradiação gama. Desta forma, além da caracterização inicial do fármaco ao chegar à fábrica, é importante avaliar se o processo produtivo também pode alterar a forma polimorfa. Segundo Aulton, no decorrer do prazo de validade de um medicamento pode haver a reversão de formas metaestáveis, se utilizadas, para a forma estável. ${ }^{53}$

O DSC apresenta ainda a capacidade de distinguir entalpicamente entre as formas cristalinas, e pode ser uma ferramenta fundamental na identificação da forma estável e das formas metaestáveis do fármaco.

\section{Cinética de degradação térmica}

A cinética de degradação térmica é utilizada para avaliação de estabilidade de fármacos e de formulações farmacêuticas e pode ser determinada tanto por TG dinâmica quanto por TG isotérmica. Tanto a cinética de degradação não isotérmica quanto a cinética de degradação isotérmica são utilizadas na área farmacêutica, no entanto, em estudos isotérmicos existe a possibilidade de se determinar o prazo de validade por extrapolação, utilizando a Equação de Arrhenius. Alguns autores relataram estudos de cinética na área de fármacos, como Barreto Gomes et al.; Macedo et al.; Macedo e Nascimento; Rodrigues et al.; Souza et al.; Medeiros et al.; Souza et al.; Souza et al. e Nunes et al. ${ }^{24,26,54-60}$

Barreto Gomes et al. correlacionaram a cinética de degradação térmica com a cinética de dissolução da ampicilina. Os estudos foram conduzidos sob cinética de degradação isotérmica e a constante de velocidade de degradação térmica $(\mathrm{k})$ foi comparada com a velocidade de dissolução, observando-se uma relação entre as duas constantes. ${ }^{54}$

Macedo et al. relataram a existência de interação entre lactose e propranolol nas formulações de comprimidos, utilizando as técnicas de TG e DSC fotovisual. Apresentaram dados cinéticos comparativos como a constante de velocidade de degradação, obtidos a partir de TG isotérmicas, demonstrando uma menor estabilidade das formulações em relação ao fármaco..$^{55}$

Macedo e Nascimento relataram a cinética de degradação térmica e estudos de compatibilidade de formulação do tiabendazol. O estudo foi conduzido pelo método isotérmico. Os autores relataram que a degradação segue o modelo cinético de primeira ordem e que a formulação tem maior velocidade de degradação em relação ao fármaco, e justificam que isto pode ser devido à presença de maior quantidade de água na formulação. ${ }^{24}$

Rodrigues et al. relataram a cinética de degradação isotérmica do fármaco zidovudina, com prazo de validade de 427 dias calculado por extrapolação utilizando a Equação de Arrhenius. ${ }^{26}$

Souza et al. relataram que existe alguma incompatibilidade nas formulações de metronidazol; utilizando cinética de degradação isotérmica demonstraram que as formulações do mercado apresentam maior velocidade de degradação e menor estabilidade, em relação ao fármaco..$^{56}$

Medeiros et al. relataram incompatibilidades nas formulações de prednisona do mercado brasileiro, demonstrando que as formulações apresentam menor estabilidade em relação ao fármaco. Os estudos foram realizados por DSC e cinética de degradação isotérmica por TG, onde foram comparadas as velocidades de degradação. ${ }^{57}$

Souza et al. relataram a correlação entre a estabilidade da formulação e a dissolução para a cimetidina. O estudo foi realizado com cinética de degradação isotérmica e os autores concluíram que as formulações mais estáveis têm melhor desempenho na dissolução do fármaco. ${ }^{58}$

Souza et al. relataram a estabilidade e cinética de degradação dos fármacos lovastatina e sinvastatina. Concluíram que a lovastatina é mais estável que a sinvastatina, apresentando os valores de Ea, com valores superiores para a lovastatina. O estudo foi realizado tanto em atmosfera inerte quanto oxidativa, e o modelo matemático que foi ajustado para os fármacos foi o modelo de degradação F1, que representa a degradação segundo o modelo cinético de primeira ordem. ${ }^{59}$

Nunes et al. relataram a compatibilidade do cloridrato de verapamil frente a alguns excipientes geralmente utilizados em formulação de comprimidos. Demonstraram não haver problemas de incompatibilidade utilizando DSC em análise de misturas binárias (fármaco-excipientes) e determinaram a cinética de degradação pelo método não isotérmico de Ozawa. ${ }^{60}$

\section{RELEVÂNCIA}

A qualidade, a segurança e a eficácia dos produtos farmacêuticos são uma preocupação econômico-social constante dos órgãos sanitários de todos os países. O setor público é responsável pela definição dos parâmetros de qualidade dos medicamentos, bem como pela fiscalização do seu cumprimento. É obrigação da indústria farmacêutica o cuidado rigoroso com a qualidade e segurança dos seus processos e produtos. $\mathrm{O}$ fornecimento de especificações mínimas de qualidade para os produtos farmacêuticos e para todos os insumos utilizados na sua fabricação é de competência legal e exclusiva das farmacopeias. As especificações das farmacopeias regulam as relações com o comércio exterior (importação e exportação), como os requisitos mínimos de qualidade de insumos e produtos farmacêuticos produzidos no país, servindo, ainda, como parâmetros para as ações de vigilância sanitária. ${ }^{61}$

Segundo as instruções da agência americana FDA, para manter a qualidade do produto farmacêutico com a mesma segurança e eficácia terapêutica em relação ao medicamento referência (o qual tem todos os estudos clínicos evidenciados), o princípio ativo farmacêutico deve apresentar, além dos testes exigidos nas farmacopeias, especificações internas da indústria, quanto à existência ou ausência de polimorfismo, tamanho de partícula e solubilidade. Dentre as técnicas analíticas utilizadas para determinar o polimorfismo destaca-se em importância a análise térmica, por meio de DSC, TG e DTA. ${ }^{62,63}$

Algumas substâncias existem sob diferentes formas cristalinas, o que é chamado de polimorfismo, e que diferem em propriedades físico-químicas. $\mathrm{O}$ polimorfismo também pode incluir produtos solvatados ou hidratados (pseudopolimorfos) e a forma amorfa. Este fenômeno pode levar a alterações nos produtos farmacêuticos no que se refere a parâmetros de pureza, estabilidade, qualidade ou eficiência e biodisponibilidade do produto farmacêutico, o que acarreta o comprometimento do efeito in vivo. ${ }^{63-66}$

Polimorfos diferentes variam quanto às suas propriedades físicas e químicas, como a dissolução e a estabilidade do estado sólido, assim como quanto ao seu comportamento tecnológico no que diz respeito, em alguns casos, ao fluxo do pó e à compatibilidade durante a compressão. Sob condições de temperatura e pressão definidas, apenas uma das possíveis formas polimórficas de uma substância pura é estável, sendo as outras formas denominadas metastáveis, formas que se transformam, a diferentes velocidades, na forma estável. As formas polimórficas metaestáveis apresentam maior velocidade de dissolução e, consequentemente, maior biodisponibilidade..$^{12,53}$

Além do polimorfismo, outras questões de âmbito farmacêutico demonstram a relevância da análise térmica para o futuro da indústria farmacêutica, são elas: a caracterização dos fármacos com seus eventos térmicos definidos; estudos de pureza de fármacos realizados 
por TG e DSC; estudos de compatibilidade por DSC, que têm sido largamente utilizados nas últimas décadas como uma ferramenta para avaliação de pré-formulações e, definição da estabilidade dos fármacos e da formulação farmacêutica, acarretando até mesmo em definições sobre as condições de armazenamento do medicamento. Giron, Brittain e Craig e Reading relataram aplicações da análise térmica na área farmacêutica e apresentaram alguns exemplos. ${ }^{9,12,20,25}$

\section{METODOLOGIA}

\section{Caracterização}

As curvas TG são usualmente obtidas com razão de aquecimento de $10{ }^{\circ} \mathrm{C} \mathrm{min}^{-1}$, iniciando-se à temperatura ambiente até $750{ }^{\circ} \mathrm{C}$, em atmosfera dinâmica de nitrogênio e com vazão de $50 \mathrm{~mL} \mathrm{~min}{ }^{-1}$, cadinho de alumina e massa de amostra de aproximadamente 5,0 mg.

As curvas DSC são usualmente obtidas sob atmosfera dinâmica de nitrogênio com vazão de $50 \mathrm{~mL} \mathrm{~min}^{-1}$, razão de aquecimento de $10^{\circ} \mathrm{C} \mathrm{min}^{-1}$, com aquecimento desde a temperatura ambiente até 400 ${ }^{\circ} \mathrm{C}$, cadinho de alumínio parcialmente fechado e massa de amostra em torno de $0,5 \mathrm{mg}$. O equipamento deve ser previamente calibrado com índio (PF 156,6 ${ }^{\circ} \mathrm{C} ; \Delta$ Hfusão = 28,54 J g ${ }^{-1}$ ) e chumbo (PF 327,0 ${ }^{\circ} \mathrm{C}$ ), ou outros padrões recomendados pelo fabricante. A avaliação de pureza por DSC é realizada aplicando-se a Equação de van't Hoff ao pico de fusão do fármaco.

\section{Compatibilidade de formulação farmacêutica}

Os estudos de compatibilidade das formulações farmacêuticas são realizados utilizando a técnica de DSC. Os excipientes listados para o desenvolvimento dos comprimidos, o fármaco, as misturas binárias 1:1 do fármaco com cada excipiente, a formulação teste (mistura multicomponente) e o placebo são submetidos a ensaios por DSC para se avaliar o comportamento térmico dos fármacos e a compatibilidade das formulações farmacêuticas.

$\mathrm{Na}$ avaliação de compatibilidade por DSC, uma interação pode ser visualizada como uma mudança no ponto de fusão, na forma, na área de picos, no aparecimento de uma transição, aparecimento ou desaparecimento de picos após mistura de componentes. No entanto, após mistura binária de dois componentes há invariavelmente alguma alteração na temperatura de transição, na forma e área dos picos, e isso pode não ser uma interação danosa e deve ser interpretado com cautela. Se o excipiente é reativo do ponto de vista químico e incompatível com o fármaco, este deve ser evitado. Quando se suspeita de uma reação química e/ou interação, mas as mudanças térmicas forem pequenas, a incompatibilidade deve ser confirmada por outras técnicas analíticas, como a CLAE. A vantagem do DSC sobre os métodos rotineiros de pesquisa de compatibilidade consiste em não ser necessário o armazenamento por longos períodos da mistura antes da avaliação, sob condições extremas de temperatura e umidade para acelerar as interações, como recomendado pela legislação vigente. , $^{8,930,53}$

A pesquisa de interações fármaco-excipiente em mistura binária é feita inicialmente com misturas binárias utilizando-se $5 \mathrm{mg}$ de fármaco em uma mistura de $50 \%$ com o adjuvante, para maximizar a probabilidade de se observar uma interação; e, em seguida, é avaliada a mistura multicomponente. ${ }^{32,34,67}$

É importante observar que existe uma alteração no valor da entalpia de fusão do fármaco quando associado aos excipientes (mistura física). Este valor equivale a praticamente a metade do valor de $\Delta \mathrm{H}$ do fármaco, visto que se trata de misturas físicas na proporção 1:1. Para que não seja caracterizada uma incompatibilidade, os eventos térmicos mais significativos devem ocorrer nas mesmas faixas de temperatura. Porém, pequenas alterações podem estar relacionadas à presença dos excipientes na formulação, porém, podem não caracterizar a ocorrência de interações ou reação química. ${ }^{39,68}$

Em uma análise por DSC de mistura binária, ou multicomponente, pode ser que exista a solubilização do fármaco durante o aquecimento por um excipiente da mistura; isto acontece quando o excipiente tem menor faixa de fusão e consegue solubilizar o fármaco presente numa mistura. No entanto, se existem cristais do fármaco intactos, este funde na faixa adequada e por isso não existe interferência nas análises de compatibilidade propostas. Uma adequação da taxa de aquecimento pode ser necessária para melhor visualizar a faixa de fusão do fármaco nestas condições onde existe solubilização do fármaco, e uma alternativa seria um aquecimento com alto valor de razão de aquecimento. Para visualizar melhor a solubilização do fármaco pelo excipiente, algumas alternativas são possíveis, como a análise termo óptica (TOA) ou o uso de DSC fotovisual.

Para a avaliação mais minuciosa de uma interação de um fármaco com um excipiente, já identificada na análise por DSC, é possível executar um estudo por TG dinâmico. É realizada uma curva TG dinâmica para o fármaco, outra curva para o excipiente específico, uma terceira referente à mistura binária. Se o resultado da curva TG dinâmica da mistura binária não for o somatório das curvas TG individuais do fármaco mais o excipiente, existe incompatibilidade na mistura proposta.

\section{Pesquisa de cristais}

Quanto à avaliação de presença de polimorfismo nos fármacos por DSC, a dificuldade inicial é medir o ponto de fusão da(s) forma(s) metaestável(is) (instáveis); a velocidade de aquecimento é crítica neste ponto. Um aquecimento muito rápido pode ocultar uma curva endotérmica, enquanto um aquecimento muito lento pode permitir uma transição ou decomposição, dificultando a visualização dos eventos térmicos.

A pesquisa de cristais é executada, inicialmente, por meio de um estudo em duas razões de aquecimento, 2 e $20^{\circ} \mathrm{C} / \mathrm{min}$, com o objetivo de identificar múltiplos picos de fusão e eventos de transição cristalina para todos os lotes de cada fármaco.

Em seguida, o fármaco pode ser recristalizado sob condições variadas de solventes, tais como, diclorometano, metanol, etanol, água, acetona e hexano; temperatura: ambiente e resfriado a $-10^{\circ} \mathrm{C}$; e grau de saturação: saturado e diluído. Os cristais obtidos são submetidos a análises por DSC, TOA (análise termo-óptica), microscopia óptica acoplada à câmera fotográfica e DRX.

A TOA é um método que permite o acompanhamento das alterações produzidas numa amostra por variação de temperatura através de observação microscópica com luz polarizada. Este método é de grande utilidade e é complementar ao DSC para informações de compatibilidade de formulações e identificação de polimorfismo. ${ }^{65}$

No caso de um polimorfismo farmacêutico, o resultado de uma curva DSC é o aparecimento de múltiplos picos de fusão relacionados a cada forma cristalina. O polimorfismo pode ser identificado com o somatório de algumas técnicas, ou seja, na análise por DRX pode-se observar a presença de picos de difração em ângulos distintos para cada forma cristalina; na curva DSC, com a presença de picos de fusão característicos de cada forma; na microscopia óptica com a visualização das diferentes formas espaciais dos cristais e, na análise por infravermelho (IV) pelo deslocamento de bandas características derivadas da interação intermolecular diferente de cada forma cristalina.

A DSC é a única técnica analítica que apresenta correlação com a estabilidade das formas cristalinas. Uma relação monotrópica entre dois cristais é estabelecida quando existe uma transição cristalina (Tc) exotérmica, a forma cristalina de maior faixa de fusão apresenta um maior calor de fusão ( $\Delta \mathrm{Hf})$, sendo esta a forma cristalina estável. Quando uma relação enantiotrópica entre dois cristais é estabelecida, 
existe uma transição cristalina (Tc) endotérmica, a forma cristalina de maior faixa de fusão apresenta um menor calor de fusão $(\Delta \mathrm{Hf})$, sendo esta a forma cristalina metaestável.

Florence e Attwood e Brittain relataram que dois cristais podem se apresentar com morfologias diferentes e com a mesma cela unitária; isto pode acontecer por influência no eixo de crescimento do núcleo, que é dependente de concentração, temperatura e tipo de solventes utilizados. ${ }^{12,69}$

Desta forma, em alguns casos, o polimorfismo se encontra de forma diferenciada. Ou seja, na análise por microscopia óptica é possível observar a presença do polimorfismo; em curvas DSC não são identificados múltiplos picos de fusão, enquanto na análise por DRX não é possível observar diferenças cristalográficas entre as formas cristalinas. No entanto, no DRX se observam diferenças na intensidade, em planos específicos de reflexão, o que sugere cristais de mesma cela unitária com orientação preferencial para o desenvolvimento macroscópico dos cristalitos de formas diferentes.

Outra observação possível com a análise térmica, com o uso do DSC junto ao equipameto de DRX, pode ser em processos de amorfisação de fármacos na presença de alguns excipientes, conforme relatado por Moneghini et al. para o fármaco atenolol e na patente do fármaco sinvastatina (Sundaram et al), onde os autores relataram a amorfização na presença de povidona. ${ }^{41,70}$

\section{Cinética de degradação do fármaco e da formulação farmacêutica}

A avaliação da estabilidade do fármaco e da formulação farmacêutica pode ser realizada por estudos de cinética de degradação isotérmica com extrapolação do prazo de validade a $25^{\circ} \mathrm{C}$ por meio da Equação de Arrhenius. Os estudos de cinética são realizados da seguinte maneira: A. A partir das curvas TG dinâmica do fármaco e da formulação farmacêutica são obtidas curvas do tipo TG isotérmica nas temperaturas de início do processo de degradação, em atmosfera de nitrogênio.

B. A ordem da reação de degradação é estabelecida avaliando-se os ajustes dos modelos de zero, primeira e segunda ordens através do coeficiente de correlação linear (r). Com o recurso da Equação de Arrhenius é possível calcular a constante de velocidade da reação $(\mathrm{k})$ a $25^{\circ} \mathrm{C}$ por extrapolação e, diante deste valor, calculase o prazo da validade, $\mathrm{t} 90$, na temperatura de $25^{\circ} \mathrm{C}$. O prazo de validade, t90, representa o tempo necessário para a concentração do fármaco atingir $90 \%$ da concentração inicial, e é aceito como determinação de prazo de validade.

As equações utilizadas para descrever a cinética de degradação isotérmica, e relatadas por vários autores, podem ser visualizadas a seguir. $^{24,26,34,36,55-58,71}$

Quadro 1. Equações utilizadas para descrever a cinética de degradação isotérmica

\begin{tabular}{|c|c|c|}
\hline Ordem zero & Primeira ordem & Segunda ordem \\
\hline $\mathrm{C}=-k \mathrm{~T}+\mathrm{C}_{\mathrm{o}}$ & $\log \mathrm{C}=-\frac{k \mathrm{~T}}{2,303}+\log \mathrm{C}_{\mathrm{o}}$ & $\frac{1}{\mathrm{C}}=k \mathrm{~T}+\frac{1}{\mathrm{C}_{\mathrm{o}}}$ \\
\hline$t_{90}=\frac{0,1 \times C_{\mathrm{o}}}{k}$ & $t_{90}=\frac{0,105}{k}$ & $t_{90}=\frac{1}{9 \times k \times C_{o}}$ \\
\hline
\end{tabular}

$\mathrm{C}=$ concentração $/ \% ; k=$ constante de velocidade $\left(\mathrm{s}^{-1}\right) ; \mathrm{Co}=$ concentração inicial; $\mathrm{t}=$ tempo $/ \mathrm{s}$

\section{Equação de Arrhenius (2):}

$$
\log K=-\frac{E_{a}}{2,303 R} \times \frac{1}{T}+\log A
$$

onde: $k=$ constante de velocidade; $\mathrm{Ea}=$ energia de ativação; $\mathrm{A}=$ fator pré exponencial; $\mathrm{T}=$ temperatura em Kelvin e $\mathrm{R}=$ constante universal dos gases $\left(8,314 \mathrm{~J} \mathrm{~mol}^{-1} \mathrm{~K}^{-1}\right)$.

$\mathrm{O}$ método gráfico da Equação de Arrhenius, 1/T versus $\log \mathrm{k}$ pode ser mais facilmente entendido. A inclinação da reta é definida por $\mathrm{Ea} /(\mathrm{R} \times 2,303)$, onde a energia de ativação pode ser calculada multiplicando-se a inclinação pela constante universal dos gases $\mathrm{R}$ $\left(8,314 \mathrm{~J} \mathrm{~mol}^{-1} \mathrm{~K}^{-1}\right)$ e por 2,303 . A Ea (energia de ativação) também expressa a estabilidade do fármaco ou da formulação farmacêutica em questão, sendo diretamente proporcional à estabilidade.

\section{CONCLUSÕES}

A análise térmica mostra-se como uma ferramenta poderosa na área farmacêutica. O uso da técnica no desenvolvimento das formulações farmacêuticas, evidenciando possíveis incompatibilidades; na avaliação da estabilidade dos fármacos e das formulações farmacêuticas, prevendo prazos de validade e definindo estratégias para acondicionamento dos fármacos e, na pesquisa de polimorfos, caracterizando e identificando formas cristalinas, tudo isto demonstra a importância da técnica no cotidiano de uma indústria farmacêutica para garantir a qualidade do produto final.

\section{REFERÊNCIAS}

1. Aulton, M. E.; Delineamento de formas farmacêuticas, $2^{\mathrm{a}}$ ed.; ARTMED: Porto Alegre, 2005.

2. Balestrieri, F.; Magri, A. D.; Magri, A. L.; Marini, D.; Sacchini, A.; Thermochim. Acta 1996, 285, 337.

3. Barreto Gomes, A. P.; Souza, F. S.; Macedo, R. O.; J. Therm. Anal. Calorim. 2006, 72, 545.

4. Bazzo, G. C.; Segatto Silva, G. C.; Braz. J. Pharm. Sci. 2005, 41, 315.

5. Bergese, P.; Bontempi, E.; Colombo, I.; Gervasoni, D.; Depero, L. E.; Compos. Sci. Technol. 2003, 63, 1197.

6. Brasil, Agência Nacional de Vigilância Sanitária, Resolução RDC n ${ }^{\circ}$ 150, 17/6/2003, Diário Oficial da União, Poder Executivo, Brasília, DF, 20/06/2003.

7. Brasil, Agência Nacional de Vigilância Sanitária, Resolução RDC n 199, 12/7/2002, Diário Oficial da União, Poder Executivo, Brasília, DF, 17/07/2002.

8. Brasil, Agência Nacional de Vigilância Sanitária, Resolução RE n ${ }^{\circ}$, 29/7/2005, Diário Oficial da União, Brasília, DF, 01/08/2005.

9. British Pharmacopeia 2007; version 11.0 [Cd-Rom], The Stationery Office: London, 2007.

10. Brittain, H. G.; Polymorphism in Pharmaceutical Solids, Marcel Dekker: New York, 1999.

11. Brown, M. E.; Introduction to Thermal Analysis: Techniques and Applications, $2^{\text {nd }}$ ed.; Kluwer Academic Publishers: New York, 2001.

12. Caira, M. R.; Foppoli, A.; Sangalli, M. E.; Zema, L.; Giordano, F.; J. Therm. Anal. Calorim. 2004, 77, 653.

13. Carstensen, J. T.; Advanced Pharmaceutical Solids, Marcel Dekker: New York, 2001

14. Ceschel, G. C.; Badiello, R.; Ronchi, C.; Maffei, P.; J. Pharm. Biomed. Anal. 2003, 32, 1067.

15. Cides, L. C. S.; Araújo, A. A. S.; Santos-Filho, M.; Matos, J. R.; J. Therm. Anal. Calorim. 2006, 84, 441.

16. Craig, D. Q. M.; Reading, M.; Thermal Analysis of Pharmaceuticals, CRC Press: New York, 2007.

17. Dorothee-Sophie, C.; Dalton, C. R.; Hancock, B. C.; PSTT Res. Focus 1999, 2, 311 .

18. European Pharmacopoeia, $6^{\text {th }}$ ed.; European Department for Quality of Medicines: Strasbourg, 2007. 
19. Farmacopéia Portuguesa, VII ed.; Imprensa Nacional Casa da Moeda: Lisboa, 2002.

20. Ferguson, H. F.; Frurip, D. J.; Pastor, A. J.; Peerey, L. M.; Whiting, L. F.; Thermochim. Acta 2000, 363, 1.

21. Florence, A. T.; Attwood, D.; Princípios físico químicos em Farmácia, $1^{\mathrm{a}}$ ed.; EDUSP: São Paulo, 2003.

22. Freitas, M. N.; Alves, R.; Matos, J. R.; Marchetti, J. M.; J. Therm. Anal. Calorim. 2007, 87, 905.

23. Gabbott, P.; Principles and applications of thermal analysis, Blackwell Publishing: Oxford, 2008.

24. Gasparotto, F. S.; Dissertação de Mestrado, Universidade Federal do Rio Grande do Sul, Brasil, 2005, disponível em http://servicos. capes.gov.br/arquivos/avaliacao/estudos/ dados1/2005/42001013/019/ 2005_019_42001013087P7_Teses.pdf, acessada em Agosto 2010.

25. Giordano, F.; Rossi, A.; Pasquali, I.; Bettini, R.; Frigo, E.; Gazzaniga, A.; Sangalli, M. E.; Mileo, V.; Catinela, S.; J. Therm. Anal. Calorim. 2003, 73, 509 .

26. Giron, D.; J. Therm. Anal. Calorim. 2002, 68, 335.

27. Giron, D.; J. Therm. Anal. Calorim. 1999, 56, 1285.

28. FDA, Department of Health and Human Services, Center for Veterinary Medicine; Guidance for Industry Specifications: Test Procedures And Acceptance Criteria For New Veterinary Drug Substances And New Medicinal Products: Chemical Substances - Draft Guidance, May, 2005, disponível em http://www.fda.gov/cvm/default.html, acessada em Julho 2010.

29. Food and Drug Administration, Center for Drug Evaluation and Research; Guidance for industry: ANDAs: Pharmaceutical Solid Polymorphism: Chemistry, Manufacturing, and Controls Information, July, 2007, disponível em http://www.fda.gov/downloads/Drugs/ GuidanceComplianceRegulatoryInformation/Guidances/ucm072866.pdf, acessada em Julho 2010.

30. Food and Drug Administration, Center for Drug Evaluation and Research; Guidance for industry: Waiver of in vivo bioavailability and bioequivalence studies for immediate relase solid oral dosage forms based on a biopharmaceutics classification system, August, 2000.

31. Food and Drug Administration, Center for Drug Evaluation and Research; Guidance: Submitting Supporting Documentation in Drug Applications for the Manufacture of Drug Substances, February, 1987, disponível em http://www.thecre.com/fedlaw/ legal17/guidance.htm, acessada em Julho 2010.

32. Hassan, M. A.; Salem, M. S.; Sueliman, M. S.; Najib, N. M.; Int. J. Pharm. 1997, 149, 227.

33. Hatakeyama, T.; Quinn, F. X.; Thermal analysis: Fundamentals and applications to polymer science, $2^{\text {nd }}$ ed.; John Wiley \& Sons: New York, 1999.

34. ICH Q6A - International Conference on Harmonization of Technical Requirements for the Registration of Drugs for Human Use; Specifications: test procedures and acceptance criteria for new drug substances and new drug products: chemical substances Q6A, October, 1999, disponível em http://www.ich.org/LOB/media/MEDIA430.pdf, acessada em Junho 2010.

35. Japanese Pharmacopeia, XIV ed.; 2001, disponível em http://jpdb.nihs. go.jp/jp14e/, acessada em Fevereiro 2010.

36. Kim, C.; Advanced pharmaceutics: physicochemical principles, CRC Press: New York, 2004.

37. Laszcz, M.; Kosmacinska, B.; Korczak, K.; Smigielska, B.; Glice, M.; Maruszak, W.; Groman, A.; Beczkowicz, H.; Zelazko, L.; J. Therm. Anal. Calorim. 2007, 88, 305

38. Leitão, M. L. P.; Canotilho, J.; Cruz, M. S. C.; Pereira, J. C.; Souza, A. T.; Redinha, J. S.; J. Therm. Anal. Calorim. 2002, 68, 397.

39. Macedo, R. O.; Nascimento, T. G.; Thermochim. Acta 2002, 392-393, 85.

40. Macedo, R. O.; Nascimento, T. G.; J. Therm. Anal. Calorim. 2001, 64, 751.
41. Macedo, R. O.; Nascimento, T. G.; Aragão, C. F. S.; Barreto Gomes, A. P.; J. Therm. Anal. Calorim. 2000, 59, 657.

42. Macedo, R. O.; Nascimento, T. G.; Veras, J. W. E.; J. Therm. Anal. Calorim. 2001, 64, 757.

43. Macedo, R. O.; Nascimento, T. G.; Veras, J. W. E.; J. Therm. Anal. Calorim. 2002, 67, 483.

44. Marciniec, B.; Kozak, M.; Wachowski, L.; Ogrodowczyk, M.; J. Therm. Anal. Calorim. 2003, 73, 473.

45. Mcgregor, C.; Saunders, M. H.; Buckton, G. R. D.; Saklatvala, R. D.; Thermochim. Acta 2004, 417, 231.

46. Medeiros, A. C. D.; Cervantes, N. A. B.; Gomes, A. P. B.; Macedo, R. O.; J. Therm. Anal. Calorim. 2001, 64, 745.

47. Moneghini, M.; Carcano, A.; Zingone, G.; Perissutti, B.; Int. J. Pharm. 1998, 175, 177.

48. Mura, P.; Faucci, M. T.; Manderioli, A.; Bramanti, G.; Ceccarelli, L.; J. Pharm. Biomed. Anal. 1998, 18, 151.

49. Nunes, R. S.; Semaan, F. S.; Riga, A. T.; Cavalheiro, E. T. G.; J. Therm. Anal. Calorim. 2009, 97, 349.

50. Oliveira, G. G. G.; Ferraz, H. G.; Matos, J. S. R.; J. Therm. Anal. Calorim. 2005, 79, 267.

51. Palacio, M. A.; Cuffini, S.; Bandini, R.; Karlsson, A.; Palacios, S. M.; J. Pharm. Biomed. Anal. 2007, 43, 1531.

52. Porter III, W. W.; Elie, S. C.; Matzger, A. J.; Cryst. Growth Des. 2008, 8,14 .

53. Reis, A. M. M.; Resende, D. C.; Moreira-Campos, L. M.; Teste de controle de qualidade de medicamentos. SBRAFH: Belo Horizonte, 1999.

54. Rodrigues, P. O.; Cardoso, T. F. M.; Silva, M. A. S.; Matos, J. R.; Acta Farm. Bonaer. 2005, 24, 383.

55. Rodrigues-Spong, B.; Price, C. P.; Jayasankar, A.; Matzger, A. J.; Rodrigues-Homedo, N. R.; Adv. Drug Delivery Rev. 2004, 56, 241.

56. Roux, M. V.; Davalos, J. Z.; Jimenez, P.; Thermochim. Acta 2002, 394, 19.

57. Rustichelli, C.; Gamberini, G.; Ferioli, V.; Gamberini, M. C.; Ficarra, R.; Tommasini, S.; J. Pharm. Biomed. Anal. 2000, 23, 41.

58. Sacchetti, M.; J. Therm. Anal. Calorim. 2001, 63, 345.

59. Schnitzler, E.; Carvalho-Filho, M. A. S.; Stadler, C. C.; Volpato, A. M.; Ionashiro, M.; Eclética Química On-Line 2001, 26, 71.

60. Souza N. A. B.; Medeiros, A. C. D.; Santos, A. F. O.; Macedo, R. O.; J. Therm. Anal. Calorim. 2003, 72, 535.

61. Souza, F. S.; Basilio Jr, I. D.; Oliveira, E. J.; Macedo, R. O.; J. Therm. Anal. Calorim. 2003, 72, 549.

62. Souza, M. A. F.; Conceição, M. M.; Silva, M. C. D.; Soledade, L. E. B.; Souza, A. G.; J. Therm. Anal. Calorim. 2007, 87, 859.

63. Stulzer, H. K.; Rodrigues, P. O.; Cardoso, T. M.; Matos, J. S. R.; Silva, M. A. S.; J. Therm. Anal. Calorim. 2008, 91, 323.

64. Sundaram, V.; Kharkar, M. R.; Yarraguntla, S. R.; Gudipati, S.; Mandava, V. N. B. R.; US pat 2006/0223882 A1, 2006.

65. Maria, T. M. R.; Nunes, R. M. D.; Pereira, M. M.; Eusébio, M. E. S.; Quim. Nova 2009, 32, 2225.

66. Dias, A. G.; Soares, R. O.; Quim. Nova, 2009, 32, 2230.

67. Swanepoel, E.; Liebenberg, W.; Villiers, M. M.; Eur. J. Pharm. Biopharm. 2003, 55, 345.

68. The Merck Index; version 12:3 [Cd-Rom], Chapman \& Hall: New York, 2000.

69. United States Pharmacopoeia, $31^{\text {th }}$ ed.; United States Pharmacopeial Convention: Rockville, 2008.

70. Wendlandt, W. W.; Thermal Analysis, $3^{\text {rd }}$ ed.; John Wiley \& Sons: New York, 1986

71. WHO - World Heath Organization; Quality of Active Pharmaceutical Ingredients (APIs), Workshop on GMP and Quality Assurance of HIV products, Mehmandoust, M.; PhD, consultant to WHO Agence Française de Sécurité Sanitaire des Produits de Santé (AFSSAPS), Shanghai, China, March, 2005. 